\title{
AVALIAÇÃo dA CONCENTRAÇÃO DE METAIS PESAdOS EM TECIDO DE OSTRAS Crassostrea gigas (MOLUSCA, BIVALVE) CULTIVADAS NA BAÍA DA BABITONGA, LITORAL NORTE DE SANTA CATARINA
}

\author{
CLÁUDIO RUDOLFO TURECK* \\ THEREZINHA MARIA NOVAIS DE OLIVEIRA** \\ MARTA JUSSARA CREMER $R^{\star * *}$ \\ JACKSON CESAR BASSFELD***
}

\begin{abstract}
Este trabalho objetivou avaliar a concentração de metais pesados em tecidos de ostras Crassostrea gigas cultivadas em 4 pontos da Baía da Babitonga, litoral Norte do Estado de Santa Catarina. Para tanto, foram instaladas estruturas de cultivo em cada ponto no sistema longline. Durante um ano foram medidos os parâmetros físico-químicos da água, quinzenalmente, e realizadas análises químicas bimensais dos metais pesados arsênio, cádmio, cobre, níquel e zinco no sedimento, na água e no tecido de ostras. Os resultados da concentração de metais pesados mostraram teores acima do permitido pela legislação brasileira para arsênio, níquel, cobre, cádmio e zinco durante o período do verão. Entretanto, foi observada a presença de concentrações significativas de zinco para todos os pontos ao longo do ano. Constatou-se que as ostras apresentaram assimilação de contaminantes, indicando a biodisponibilidade no ambiente, no entanto, também foi observada a capacidade de autodepuração por parte dos organismos.
\end{abstract}

PALAVRAS-CHAVE: MARICULTURA; Crassostrea gigas; METAIS PESADOS.

\footnotetext{
* Biólogo, mestre em Saúde e Meio Ambiente, doutorando em Aqüicultura, Universidade Federal de Santa Catarina, Professor, Departamento de Ciências Biológicas, Universidade da Região de Joinville (UNIVILLE).

** Engenheira Sanitarista, mestre e doutora em Gestão da Qualidade Ambiental, Professora, Departamento de Engenharia Ambiental, UNIVILLE.

*** Bióloga, mestre em Ecologia e Recursos Naturais, doutoranda em Zoologia, Universidade Federal do Paraná, Professora, Departamento de Ciências Biológicas, UNIVILLE.

*** Biólogo, mestre na área de Ecotoxicologia Aquática, Consultor ambiental (e-mail: carijo@onda.com.br).
} 


\section{INTRODUÇÃO}

As atividades humanas no litoral vêm gradativamente alterando as propriedades dos ecossistemas costeiros. Dentre essas podem ser mencionadas as atividades portuárias, a pesca predatória, a maricultura não-planejada, a agricultura e o desflorestamento, além da ausência de tratamento adequado para os efluentes urbanos e industriais (GOUDIE, 1989). A avaliação desses impactos, apenas recentemente vem sendo realizada. Informações referentes à qualidade da água e do sedimento marinho/ estuarino, bem como sobre a estrutura e funcionamento dos ecossistemas envolvidos, são fundamentais nesse processo. Da mesma forma, a análise econômica do grau de comprometimento dos recursos naturais e da saúde humana é de grande importância para justificar os custos das medidas de controle (GESAMP, 1990).

Há centenas de anos, a humanidade vem utilizando moluscos cultivados na alimentação. Porém, a saúde dos ecossistemas aquáticos costeiros propícios ao cultivo vem sendo comprometida. Conseqüentemente, o consumo de organismos contaminados pode colocar em risco a saúde das comunidades que se utilizam desse recurso (TURECK e OLIVEIRA, 2003).

Os moluscos são animais filtradores e acumulam toxinas e poluentes em seus tecidos, sendo por essa razão muito utilizados em pesquisas de contaminação. O processo de acúmulo de contaminantes passou a ser conhecido como bioacumulação (SPACIE e HAMELINK apud RAND e PETROCELLI,1985). Os organismos bivalves foram selecionados como indicadores ambientais por sua distribuição mundial, grande abundância, capacidade de assimilação da maioria dos poluentes e hábito sedentário (UNESCO, 1992).

A maricultura pode ser considerada como alternativa de renda significativa para as comunidades litorâneas (TURECK e OLIVEIRA, 2003). No Brasil, a maricultura vem se desenvolvendo rapidamente nas últimas décadas e representa enorme potencial a ser explorado. A ocorrência marcante de estuários ao longo da costa brasileira contribui de forma significativa para esse potencial, já que constituem locais com possibilidades para o cultivo de organismos marinhos, respeitada a capacidade de carga dos ambientes.

A baía da Babitonga, complexo estuarino situado no litoral norte de Santa Catarina, vem apresentando resultados significativos no cultivo de mexilhões, atingindo produção de 552 toneladas em 2004 (OLIVEIRA NETO, 2005). Contudo, a região sofre significativa pressão antrópica associada à presença do maior pólo industrial do estado no entorno da baía (caracterizado por atividades dos setores metalúrgico, têxtil, galvânico e de produção de PVC). A presença de contingente populacional de aproximadamente 500 mil habitantes, associada com a carência nos sistemas de tratamento de efluentes e presença de significativa atividade portuária, coloca em risco a qualidade dos produtos da maricultura local.

O presente trabalho teve por objetivo avaliar a concentração de metais pesados em tecidos de ostras (Crassostrea gigas), água e sedimento nas áreas de maricultura da Baía da Babitonga.

\section{MATERIAL E MÉTODOS}

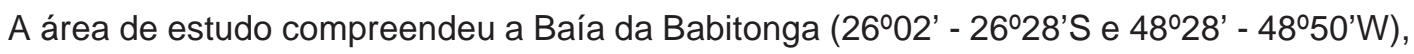
situada no extremo norte do litoral de Santa Catarina (Figura 1).

Foram selecionados 4 pontos de amostragem na baía para a instalação de cultivos experimentais de ostra (Crassostrea gigas), sendo as áreas definidas em função da existência de cultivos comercias de mexilhão (Figura 1). Os pontos escolhidos foram denominados:

Ponto 01 - FERREIRA - Associação dos Maricultores da Babitonga - AMAB (coordenadas UTM 0733138/7096685);

Ponto 02 - PAULAS - Associação de Maricultura Comunitária do Bairro do Paulas - AMACOP 
(coordenadas UTM - 0737665/7097251);

Ponto 03 - ROSA - Associação dos Maricultores do Capri - AMAPRI (coordenadas UTM - 0738796/ 7098563);

Ponto 04 - CAPRI - Associação de Aqüicultores do Balneário do Capri - AABC (coordenadas UTM 0742689/7100638).

\section{FIGURA 1 - LOCALIZAÇÃO DA BAIA DA BABITONGA NO LITORAL NORTE DE SANTA CATARINA E DOS PONTOS DE CULTIVO DE OSTRA (Crassostrea gigas), UTILIZADAS NAS AMOSTRAGENS (DATUM SAD 69 ZONA 22S)}

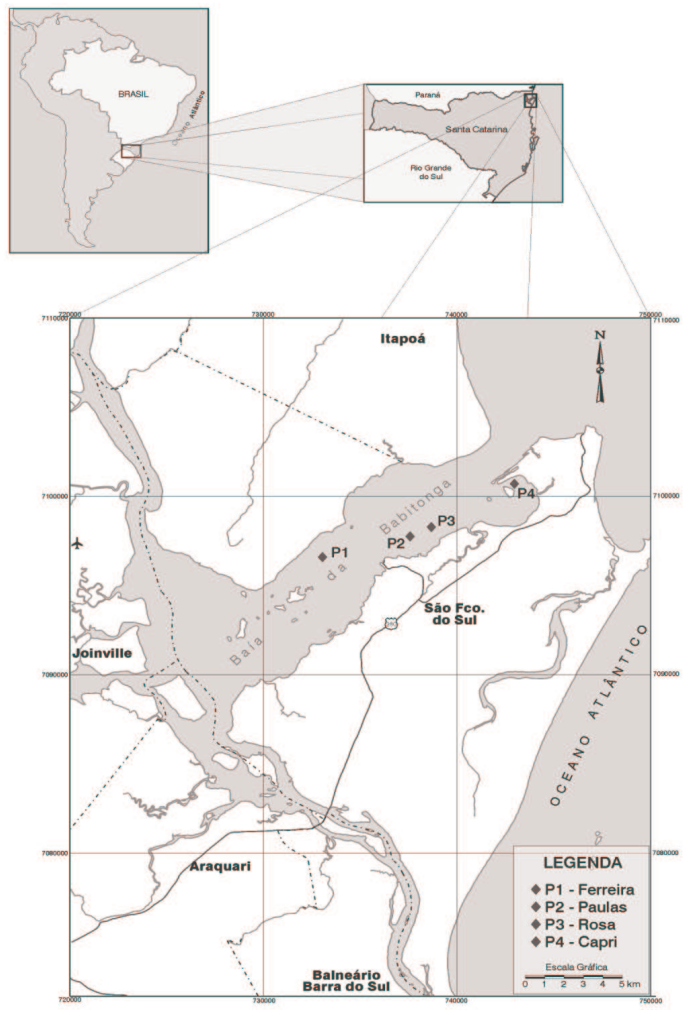

FONTE: LABORATÓRIO DE GEOPROCESSAMENTO E SENSORIAMENTO REMOTO (2002).

Os cultivos foram implantados e manejados segundo as recomendações de PEREIRA et al. (1998), utilizando o sistema do tipo "longline". Desenvolveu-se experimento no período de julho de 2000 a junho de 2001, totalizando 12 meses, sendo a montagem das estruturas padronizada para todos os pontos. As sementes de ostras Crassostrea gigas foram produzidas no Laboratório de Moluscos Marinhos da Universidade Federal de Santa Catarina (UFSC). A colocação das ostras nos pontos de amostragem ocorreu quando essas atingiram aproximadamente $5 \mathrm{~mm}$ de tamanho em altura.

Os parâmetros físico-químicos da água nos pontos de coleta foram medidos em intervalos quinzenais, avaliando-se a transparência (disco de Sechi), a temperatura na superfície (termômetro químico simples), a salinidade na superfície (refratômetro), o pH na superfície (fita indicadora) e o oxigênio dissolvido na superfície (oxímetro de campo).

A coleta de amostras para análise de metais pesados foi realizada a cada 60 dias nos quatro pontos de cultivo, considerando-se três compartimentos no ecossistema: água, sedimento e tecido de ostra, somando 6 análises para cada compartimento. Para a análise de água foram coletadas amostras 
de superfície de 3 litros, utilizando-se garrafas plásticas. No caso do sedimento foram coletadas amostras de 500 gramas, acondicionadas em sacos plásticos esterilizados, utilizando-se pegador de fundo tipo petit ponar (USEPA,1992). Para a amostragem de tecido de ostras foram coletas aproximadamente 200 gramas de acordo com as recomendações da USEPA (1992).

Foram analisados os metais pesados arsênio, cádmio, cobre, níquel e zinco conforme protocolo internacional mussel watch (UNESCO, 1992). As análises foram realizadas no Laboratório de Águas e Efluentes e Espectrometria de Absorção Atômica da Universidade Federal do Paraná (UFPR), segundo as indicações do Official Methods of Analysis of AOAC International (2000).

Os resultados das análises nos tecidos das ostras foram comparados com os parâmetros estabelecidos pela legislação para consumo de alimentos da Agência Nacional de Vigilância Sanitária (ANVISA), Portaria no 685, de 27 de agosto de 1998 (BRASIL, 1998). No caso da água, utilizou-se como parâmetro de comparação os valores estabelecidos na Resolução CONAMA n 20 (vigente na ocasião do estudo), que definia a qualidade e classificação das águas (BRASIL, 1986). Os valores encontrados nos sedimentos foram comparados com valores divulgados para o estuário de Santos, Estado de São Paulo (CETESB, 2001), em virtude da ausência de legislação específica para metais pesados em sedimentos marinhos na época.

\section{RESULTADOS E DISCUSSÃO}

\subsection{CARACTERIZAÇÃO DOS PARÂMETROS FÍSICO-QUÍMICOS}

A temperatura da água variou de $15^{\circ} \mathrm{C}$ a $28^{\circ} \mathrm{C}$ ao longo do período, considerando os quatro pontos analisados. O oxigênio dissolvido apresentou variação de $5,0 \mathrm{mg} / \mathrm{L}$ até $8,8 \mathrm{mg} / \mathrm{L}$, cujos maiores valores ocorreram nos meses de inverno.

Os valores do $\mathrm{pH}$, entre 6 a 8 ao longo do período, apresentaram pequena redução associada à diminuição da salinidade, o que pode estar relacionado aos processos de mistura das águas de diferentes origens (KUROSHIMA e BELLOTTO apud IBAMA, 1998). A salinidade apresentou comportamento similar ao longo do período de avaliação, sendo 35 o maior valor encontrado próximo a desembocadura da baía no oceano Atlântico. A diminuição dos valores de salinidade ocorreu nos meses de verão e no mês de maio, efeito associado com os maiores índices pluviométricos. O valor mínimo observado foi de 16 no mês de fevereiro (Figura 2).

\section{FIGURA 2 - VARIAÇÃO DOS VALORES DA SALINIDADE DA ÁGUA ENTRE AGOSTO DE 2000 A JUNHO DE 2001 NOS PONTOS DE CULTIVO: 1-FERREIRA, 2-PAULAS, 3-ROSA E 4-CAPRI}

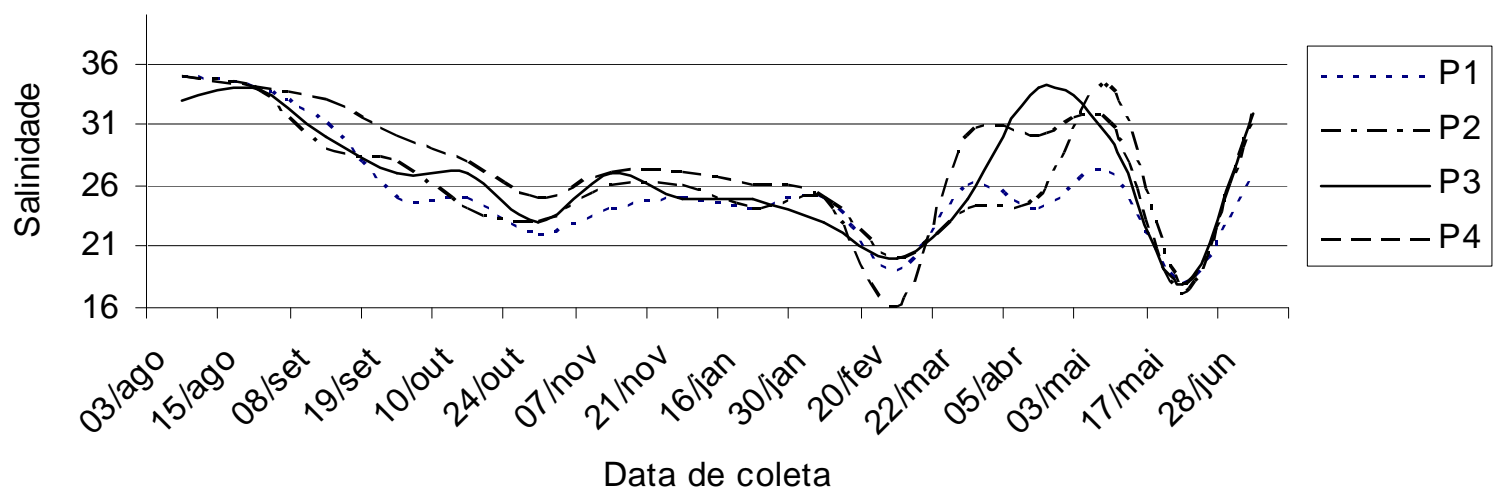




\subsection{AVALIAÇÃO DA CONTAMINAÇÃO POR METAIS PESADOS}

\subsubsection{Arsênio (As)}

A concentração de As no tecido das ostras variou de 0,17 ppm a 2,58 ppm (Tabela 1). Observouse pico de acumulação para todos os pontos no mês de fevereiro, chegando a 1,82 ppm no P2 Paulas. Após o verão verificou-se redução desse elemento para todos os pontos, associada com possível efeito de depuração das ostras, chegando a valores próximos aos permitidos pela legislação para consumo de produtos da pesca (1 ppm) (BRASIL, 1998). De acordo com a literatura os tecidos de invertebrados marinhos e peixes contém altas concentrações de arsênio, geralmente valores de 1 até 100 ppm em peso seco, sendo a maioria na forma de compostos organoarsênicos (NEFF, 1997).

O As no sedimento apresentou concentrações similares para os pontos avaliados, com exceção do P1 - Ferreira, que em janeiro evidenciou pico de 5,8 ppm. Contudo, nos meses subseqüentes tal concentração foi diminuindo gradativamente e aproximou-se dos valores encontrados para os demais pontos. O sedimento no estuário de São Vicente (SP) apresentou valores em torno de 19,9 ppm (CETESB, 2001).

A concentração de As na água comportou-se de forma similar para os quatro pontos (Tabela 1), permanecendo sempre abaixo dos limites $(0,005 \mathrm{ppm})$ permitidos pela legislação (BRASIL, 1986).

\section{TABELA 1 - VALORES DAS CONCENTRAÇÕES DE AS NA ÁGUA NOS TECIDOS DAS OSTRAS E EM SEDIMENTOS PARA OS PONTOS 1 - FERREIRA, 2 - PAULAS, 3 - ROSA E 4 - CAPRI - OUTUBRO/2000 A JUNHO/2001}

\begin{tabular}{ccccccccccccc}
\hline & \multicolumn{1}{c}{ Ponto1 } & \multicolumn{1}{c}{ Ponto 2 } & \multicolumn{1}{c}{ Arsênio (ppm) } \\
Meses & \multicolumn{1}{c}{ Ponto 3 } & \multicolumn{4}{c}{ Ponto 4 } \\
& $\mathrm{A}$ & $\mathrm{T}$ & $\mathrm{S}$ & $\mathrm{A}$ & $\mathrm{T}$ & $\mathrm{S}$ & $\mathrm{A}$ & $\mathrm{T}$ & $\mathrm{S}$ & $\mathrm{A}$ & $\mathrm{T}$ & $\mathrm{S}$ \\
Out/00 & $\mathbf{0 , 0 0 6}$ & 0,02 & 3,71 & $\mathbf{0 , 0 0 7}$ & 0,03 & 3,39 & 0,005 & 0,17 & 3,34 & 0,005 & 0,11 & 2,40 \\
Jan/01 & $<0,005$ & $\mathbf{1 , 2 9}$ & 5,78 & $<0,005$ & $\mathbf{1 , 3 5}$ & 2,45 & $<0,005$ & $\mathbf{1 , 2 9}$ & 2,58 & $<0,005$ & $\mathbf{1 , 3 6}$ & 1,84 \\
Fev/01 & $<0,010$ & $\mathbf{1 , 5 6}$ & 3,97 & $<0,010$ & $\mathbf{1 , 8 2}$ & 2,12 & $<0,010$ & $\mathbf{1 , 2 2}$ & 2,23 & $<0,010$ & $\mathbf{1 , 6 9}$ & 1,51 \\
Abr/01 & $<0,005$ & $\mathbf{0 , 8 9}$ & 3,48 & $<0,005$ & $\mathbf{1 , 0 2}$ & 2,07 & $<0,005$ & $\mathbf{1 , 0 5}$ & 2,14 & $<0,005$ & $\mathbf{1 , 0 7}$ & 1,14 \\
Mai/01 & $<0.005$ & $\mathbf{0 , 9 1}$ & 1,50 & $<0.005$ & $\mathbf{2 , 0 0}$ & 1,58 & $<0.005$ & $\mathbf{1 , 8 4}$ & 1,84 & $<0.005$ & $\mathbf{1 , 9 9}$ & 1,54 \\
Jun/01 & $<0,005$ & $\mathbf{1 , 8 7}$ & 1,73 & $<0,005$ & $\mathbf{2 , 5 8}$ & 0,89 & $<0,005$ & $\mathbf{2 , 0 5}$ & 2,18 & $<0,005$ & $\mathbf{2 , 1 4}$ & 1,44 \\
Legislação & 0,005 & 1 & - & 0,005 & 1 & - & 0,005 & 1 & - & 0,005 & 1 & - \\
\hline
\end{tabular}

$\mathrm{A}$ = água; $\mathrm{T}$ = tecido; $\mathrm{S}=$ sedimento.

Os números em negrito indicam valores acima do limite da legislação para tecido (BRASIL,1998) e água (BRASIL, 1986).

\subsubsection{Cádmio $(\mathrm{Cd})$}

A concentração de $\mathrm{Cd}$ nos tecidos das ostras variou de valores abaixo dos limites de detecção do equipamento até $3,08 \mathrm{ppm}$ (Tabela 2). Observou-se aumento na concentração para o mês de fevereiro em todos os pontos, ultrapassando os limites permitidos pela legislação de alimentos (1 ppm) (BRASIL, 1998). Após o verão ocorreu eliminação do metal nos tecidos das ostras, muito similar para os quatro pontos avaliados (valores de acordo com a legislação). No mês de junho, contudo, observaram-se concentrações próximas a 1 ppm.

O aumento das concentrações nos tecidos nas ostras coincidiu com os meses de salinidade baixa, sendo que esse fator pode aumentar a taxa de assimilação de $\mathrm{Cd}$ em organismos marinhos 
(SPACIE e HAMELINK, apud RAND e PETROCELLI, 1985). A acumulação de Cd nos tecidos dos organismos avaliados também pode estar relacionada com atividades de dragagem da área portuária. A metalização de tintas, as matérias plásticas, os pigmentos e a indústria metalúrgica são algumas aplicações do cádmio (FATMA/GTZ, 1999). No estuário de Santos (SP), os valores de Cd encontrados para os tecidos de ostras variaram de 0,8 e 0,9 ppm (CETESB, 2001).

A concentração de $\mathrm{Cd}$ no sedimento variou de valores abaixo dos limites de detecção do equipamento até $1,75 \mathrm{ppm}$ (Tabela 2). Os pontos 1 - Ferreira e 2 - Paulas apresentaram as concentrações mais altas, sendo a maior verificada no P1 - Ferreira no mês de abril (1,75 ppm). Na saída do estuário de São Vicente (SP) foram encontrados valores de 0,002 ppm de Cd no sedimento (CETESB, 2001).

A maior concentração de $\mathrm{Cd}$ na água ocorreu no mês de janeiro para os quatro pontos (Tabela 2), cujos valores sempre se mantiveram acima do permitido pela legislação $(0,005 \mathrm{ppm})$ (BRASIL, 1986). No mês de janeiro também foram constatados os mais altos valores de temperatura da água ao longo do estudo.

\section{TABELA 2 - VALORES DAS CONCENTRAÇÕES DE CD NA ÁGUA, NOS TECIDOS DAS OSTRAS E NO SEDIMENTO PARA OS PONTOS P1 - FERREIRA, P2 - PAULAS, P3 - ROSA E P4 - CAPRI - OUTUBRO/2000 A JUNHO/2001}

\begin{tabular}{ccccccccccccc}
\hline & \multicolumn{1}{c}{ Meses } & \multicolumn{1}{c}{ Ponto1 } & \multicolumn{1}{c}{ Ponto 2 } & \multicolumn{1}{c}{ Pónto 3 } & \multicolumn{1}{c}{ Ponto 4 } \\
& $\mathrm{A}$ & $\mathrm{T}$ & $\mathrm{S}$ & $\mathrm{A}$ & $\mathrm{T}$ & $\mathrm{S}$ & $\mathrm{A}$ & $\mathrm{T}$ & $\mathrm{S}$ & $\mathrm{A}$ & $\mathrm{T}$ & $\mathrm{S}$ \\
out/00 & $\mathbf{0 , 1 3}$ & 0,00 & 0,74 & $\mathbf{0 , 1 2}$ & 0,24 & 0,74 & $\mathbf{0 , 1 2}$ & 0,00 & 0,22 & $\mathbf{0 , 1 3}$ & 0,00 & 0,32 \\
jan/01 & $\mathbf{0 , 2 1}$ & 0,26 & 0,87 & $\mathbf{0 , 2 0}$ & 2,62 & 0,66 & $\mathbf{0 , 1 9}$ & 0,27 & 0,23 & $\mathbf{0 , 1 9}$ & 0,28 & 0,34 \\
fev/01 & $\mathbf{0 , 0 7}$ & $\mathbf{2 , 5 7}$ & 0,95 & $\mathbf{0 , 0 9}$ & 0,39 & 1,31 & $\mathbf{0 , 0 9}$ & $\mathbf{2 , 5 3}$ & 0,00 & $\mathbf{0 , 0 9}$ & $\mathbf{3 , 0 8}$ & 0,00 \\
abr/01 & $\mathbf{0 , 0 9}$ & 0,49 & 1,75 & $\mathbf{0 , 1 0}$ & 0,26 & 1,42 & $\mathbf{0 , 1 0}$ & 0,35 & 0,61 & $\mathbf{0 , 1 0}$ & 0,37 & 0,51 \\
mai/01 & $\mathbf{0 , 0 0}$ & 0,30 & 1,41 & 0,00 & $\mathbf{1 , 0 2}$ & 1,40 & 0,00 & 0,35 & 0,55 & 0,00 & 0,30 & 0,44 \\
jun/01 & $\mathbf{0 , 1 1}$ & 0,00 & 0,88 & $\mathbf{0 , 1 2}$ & 0,00 & 0,74 & $\mathbf{0 , 1 1}$ & 0,72 & 0,79 & $\mathbf{0 , 1 2}$ & 0,94 & 0,00 \\
Legislação & 0,005 & 1 & - & $\mathbf{0 , 0 0 5}$ & 1 & - & 0,005 & 1 & - & 0,005 & 1 & - \\
\hline
\end{tabular}

$\mathrm{A}$ = água; $\mathrm{T}$ = tecido; $\mathrm{S}=$ sedimento.

Os números em negrito indicam valores acima do limite da legislação para tecido (BRASIL, 1998) e água (BRASIL, 1986).

\subsubsection{Cobre $(\mathrm{Cu})$}

A concentração de Cu nos tecidos das ostras variou de 1,27 ppm a 53,62 ppm (Tabela 3). No mês de fevereiro os valores de $\mathrm{Cu}$ em todos os pontos ultrapassaram o limite previsto na legislação (BRASIL, 1998). Após esse período observou-se diminuição nas concentrações, retornando a valores próximos dos limites permitidos. No estuário de Santos foram constatados valores de concentração de Cu de 5,15 a 7,54 ppm nos tecidos de ostras. Já no estuário de São Vicente foram encontrados valores de 85 a 100 ppm (CETESB, 2001).

A concentração de cobre $(\mathrm{Cu})$ no sedimento compreendeu valores não-detectáveis pelo equipamento até 3,97 ppm (Tabela 3). Os valores de concentração para o P3 mantiveram-se abaixo dos encontrados para o P2, menos em junho (3,5 ppm). Os valores de concentração de Cu no sedimento para o P4 foram ainda menores que no $\mathrm{P} 3$, atingindo níveis sempre abaixo de 1,5 ppm. Os maiores valores ocorreram nos pontos 1,2 e 3 situados próximos das atividades portuárias. O óxido de cobre foi amplamente utilizado em tintas para cascos de navios ("antifouling") e vários compostos de Cu são empregados como algicidas e fungicidas. O cobre também é muito usado na indústria eletrotécnica e na produção de ligas metálicas (FATMA/GTZ, 1999). 
A concentração de Cu na água variou de valores abaixo dos limites de detecção do equipamento até 0,13 ppm (Tabela 3). O ponto 4 evidenciou a maior concentração de Cu na água.

\section{TABELA 3 - VALORES DAS CONCENTRAÇÕES DE CU NA ÁGUA, NOS TECIDOS DAS OSTRAS E EM SEDIMENTOS PARA OS PONTOS 1 - FERREIRA, 2 - PAULAS, 3 - ROSA E 4 - CAPRI - OUTUBRO/2000 A JUNHO/2001}

\begin{tabular}{ccccccccccccc}
\hline & \multicolumn{1}{c}{ Meses } & \multicolumn{1}{c}{ Ponto1 } & \multicolumn{1}{c}{ Cobre (ppm) } & \multicolumn{4}{c}{ Ponto 3 } & \multicolumn{4}{c}{ Ponto 4 } \\
& A & T & S & A & T & S & A & T & S & A & T & S \\
out/00 & 0,05 & 8,96 & 3,35 & 0,05 & 8,43 & 1,89 & 0,05 & 6,58 & 1,50 & 0,05 & 5,87 & 1,07 \\
jan/01 & 0,12 & 6,96 & 3,97 & 0,11 & 6,30 & 2,89 & 0,11 & 6,48 & 2,24 & 0,11 & 5,19 & 1,33 \\
fev/01 & 0,00 & $\mathbf{3 8 , 3 7}$ & 3,67 & 0,00 & $\mathbf{5 3 , 6 2}$ & 2,97 & 0,00 & $\mathbf{3 2 , 0 5}$ & 1,60 & 0,00 & $\mathbf{3 6 , 7 5}$ & 1,23 \\
abr/01 & 0,06 & 5,43 & 3,52 & 0,07 & 9,63 & 2,75 & 0,06 & 6,86 & 2,14 & 0,07 & 8,23 & 0,92 \\
mai/01 & 0,00 & 1,27 & 3,97 & 0,00 & 5,26 & 2,46 & 0,00 & 5,68 & 1,66 & 0,00 & 4,88 & 1,03 \\
jun/01 & 0,12 & 10,45 & 3,09 & 0,13 & 13,73 & 1,61 & 0,13 & 8,54 & 3,61 & 0,13 & 6,32 & 0,00 \\
Legislação & 0,5 & 30 & - & 0,5 & 30 & - & 0,5 & 30 & - & 0,5 & 30 & - \\
\hline
\end{tabular}

$\mathrm{A}$ = água; $\mathrm{T}$ = tecido; $\mathrm{S}$ = sedimento.

Os números em negrito indicam valores acima do limite da legislação (BRASIL, 1998).

\subsubsection{Níquel (Ni)}

A concentração de Ni nos tecidos das ostras variou entre limites abaixo dos níveis de detecção do equipamento até 7,59 ppm (Tabela 4). No mês de fevereiro os tecidos das ostras apresentaram concentrações sensivelmente acima dos valores permitidos pela legislação (5 ppm) (BRASIL, 1998). Após o verão, os valores do Ni nos tecidos atingiram níveis de acordo com a legislação. No estuário de Santos foram encontrados valores de concentração nos tecidos de ostras de 0,13 a 0,18 ppm (CETESB, 2001).

A concentração de Ni no sedimento variou de valores abaixo do limite de detecção do equipamento até 12,67 ppm (Tabela 4). No estuário de Santos foram observadas concentrações de níquel de 0,97 a 34 ppm (CETESB, 2001).

$\mathrm{Na}$ água, as concentrações de Ni variaram entre 0,17 ppm e 0,82 ppm (Tabela 4). No mês de janeiro observou-se concentração de $0,82 \mathrm{ppm}$ no P1. Em todos os pontos e meses de coleta, com exceção do mês de maio, foram verificados níveis acima do limite permitido pela legislação $(0,1 \mathrm{ppm})$ (BRASIL, 1986). O níquel (Ni), um dos cinco elementos mais abundantes, tem sido detectado em diferentes pontos da biosfera. As aplicações mais importantes do Ni envolvem a fabricação do aço inoxidável, galvanoplastia e indústria de plásticos (AZEVEDO e CHASIN, 2003).

\subsubsection{Zinco (Zn)}

A concentração de $\mathrm{Zn}$ nos tecidos das ostras evidenciou comportamento diferente dos outros metais pesados avaliados, variando de 53,08 ppm a 184,64 ppm ao longo do experimento (Tabela 5). Desde a primeira análise, os tecidos apresentaram concentrações de Zn acima do limite permitido pela legislação (50 ppm) para todos os pontos (BRASIL, 1998). As mais altas concentrações no tecido ocorreram no P1. De forma geral, a concentração de zinco nos tecidos foi diminuindo ao longo do crescimento das ostras até se aproximar dos limites permitidos pela legislação (BRASIL, 1998) no 
mês de maio. A partir desse mês ocorreu aumento nas concentrações de $\mathrm{Zn}$ nos tecidos de forma similar nos quatro pontos avaliados. No estuário de Santos foram encontrados valores de 231,4 a 296,9 ppm de zinco nos tecidos das ostras (CETESB, 2001). O zinco, importante metal traço, é essencial para o crescimento de células animais e vegetais. Sua importância está associada com a composição de certas metaloenzimas, sendo co-fator para a regulação da atividade de enzimas específicas (fosfatase alcalina e anidrase carbônica) (WOLF,1979).

\section{TABELA 4 - VALORES DAS CONCENTRAÇÕES DE Ni NA ÁGUA, NOS TECIDOS DAS OSTRAS E EM SEDIMENTOS PARA OS PONTOS 1 - FERREIRA, 2 - PAULAS, 3 - ROSA E 4 - CAPRI - OUTUBRO/2000 A JUNHO/2001}

\begin{tabular}{|c|c|c|c|c|c|c|c|c|c|c|c|c|}
\hline \multirow{3}{*}{ Meses } & \multicolumn{12}{|c|}{ Níquel (ppm) } \\
\hline & \multicolumn{3}{|c|}{ Ponto1 } & \multicolumn{3}{|c|}{ Ponto 2} & \multicolumn{3}{|c|}{ Ponto 3} & \multicolumn{3}{|c|}{ Ponto 4} \\
\hline & A & $\mathrm{T}$ & $S$ & A & $\mathrm{T}$ & $S$ & A & $\mathrm{T}$ & $S$ & A & $\mathrm{T}$ & S \\
\hline out/00 & 0,40 & 0,00 & 8,94 & 0,40 & 0,00 & 5,09 & 0,40 & 0,00 & 4,28 & 0,41 & 0,00 & 2,89 \\
\hline $\mathrm{jan} / 01$ & 0,82 & 0,83 & 11,1 & 0,77 & 0,63 & 6,38 & 0,75 & 0,97 & 5,00 & 0,73 & 0,63 & 3,81 \\
\hline $\mathrm{fev} / 01$ & 0,17 & 5,36 & 12,2 & 0,21 & 5,81 & 8,97 & 0,20 & 5,54 & 4,81 & 0,20 & 7,59 & 3,34 \\
\hline $\mathrm{abr} / 01$ & 0,34 & 2,08 & 12,6 & 0,37 & 1,77 & 8,24 & 0,36 & 1,61 & 5,54 & 0,36 & 1,49 & 2,83 \\
\hline mai/01 & 0,00 & 1,21 & 11,9 & 0,00 & 1,43 & 6,87 & 0,00 & 1,44 & 4,74 & 0,00 & 1,33 & 2,87 \\
\hline jun/01 & 0,51 & 0,00 & 7,86 & 0,55 & 0,00 & 4,18 & 0,56 & 0,00 & 6,18 & 0,56 & 0,00 & 0,00 \\
\hline Legislação & 0,1 & 5 & - & 0,1 & 5 & - & 0,1 & 5 & - & 0,1 & 5 & - \\
\hline
\end{tabular}

$\mathrm{A}$ = água; $\mathrm{T}$ = tecido; $\mathrm{S}=$ sedimento.

Os números em negrito indicam valores acima do limite da legislação para tecido (BRASIL, 1998) e água (BRASIL, 1986).

A concentração de Zn no sedimento variou de 7,23 ppm a 74,1 ppm. O sedimento no P1 apresentou os maiores valores de concentração quando comparados com os outros pontos (Tabela 5). No estuário de Santos (SP) foram encontrados de 260 a 957 ppm de zinco nos sedimentos (CETESB, 2001).

A concentração de $Z n$ na água variou de 0,1 ppm a 0,36 ppm, sendo que a legislação reporta valor máximo de 0,17 ppm (BRASIL, 1986).

\section{TABELA 5 - VALORES DAS CONCENTRAÇÕES DE Zn NA ÁGUA, NOS TECIDOS DAS OSTRAS E EM SEDIMENTOS PARA OS PONTOS 1 - FERREIRA, 2 - PAULAS, 3 - ROSA E 4 - CAPRI - OUTUBRO/2000 A JUNHO/2001}

\begin{tabular}{|c|c|c|c|c|c|c|c|c|c|c|c|c|}
\hline \multirow{3}{*}{ Meses } & \multicolumn{12}{|c|}{ Zinco (ppm) } \\
\hline & \multicolumn{3}{|c|}{ Ponto1 } & \multicolumn{3}{|c|}{ Ponto 2} & \multicolumn{3}{|c|}{ Ponto 3} & \multicolumn{3}{|c|}{ Ponto 4} \\
\hline & A & $\mathrm{T}$ & $S$ & A & $\mathrm{T}$ & S & A & $\mathrm{T}$ & $S$ & $A$ & $\mathrm{~T}$ & $S$ \\
\hline Out/00 & 0,16 & 184,64 & 74,81 & 0,16 & 171,53 & 22,30 & 0,36 & 136,36 & 22,47 & 0,18 & 116,12 & 11,27 \\
\hline Jan/001 & 0,21 & 109,75 & 37,18 & 0,21 & 92,13 & 24,66 & 0,2 & 95,29 & 18,74 & 0,20 & 87,30 & 11,12 \\
\hline Fev/001 & 0,08 & 149,80 & 43,83 & 0,10 & 131,01 & 22,92 & 0,1 & 53,08 & 18,10 & 0,10 & 67,22 & 10,88 \\
\hline Abr/001 & 0,15 & 84,08 & 56,33 & 0,16 & 70,70 & 27,49 & 0,16 & 61,44 & 21,54 & 0,16 & 69,94 & 8,47 \\
\hline Mai/001 & 0,00 & 73,22 & 48,58 & 0,00 & 77,76 & 23,62 & 0,16 & 77,07 & 19,10 & 0,00 & 76,38 & 10,03 \\
\hline Jun/001 & 0,10 & 109,55 & 33,72 & 0,12 & 115,67 & 12,33 & 0,11 & 100,29 & 26,27 & 0,12 & 86,67 & 7,23 \\
\hline Legislação & 0,17 & 50 & - & 0,17 & 50 & - & 0,17 & 50 & - & 0,17 & 50 & - \\
\hline
\end{tabular}

A = água; $\mathrm{T}$ = tecido; $\mathrm{S}$ = sedimento.

Os números em negrito indicam valores acima do limite da legislação para tecido (BRASIL, 1998) e água (BRASIL, 1986). 


\section{CONCLUSÃO}

Os resultados das análises nos tecidos das ostras mostraram, de forma geral, assimilação de metais pesados. As concentrações presentes na água e no sedimento por sua vez indicam a biodisponibilidade de contaminantes no ambiente. Possivelmente, a assimilação de metais pesados pelas ostras possa estar relacionada com os processos de ressuspensão de sedimentos contaminados, pois as maiores concentrações nos tecidos ocorreram nos pontos próximos aos locais de dragagens e no ponto próximo ao local de despejo desses sedimentos.

De maneira geral foram observados processos de eliminação de metais pesados dos tecidos das ostras em todos os pontos avaliados. A eliminação pode estar relacionada com a menor disponibilidade de contaminantes na baía. Contudo, é importante ressaltar que existem limites nas taxas de autodepuração dos organismos e que o poder cumulativo dos metais pesados representa risco à saúde das populações consumidoras de organismos contaminados.

Durante as seis análises realizadas ao longo do ano, apenas num momento a concentração de alguns metais pesados nas ostras ultrapassou os limites permitidos para o consumo.

A legislação referente a metais pesados para o consumo de alimentos marinhos é tratada de forma muito abrangente e engloba a maioria dos organismos marinhos como "peixes e produtos da pesca". Considerando as variações intrínsecas na composição dos organismos sugere-se que tais valores sejam reavaliados. Esse seria o caso, por exemplo, da alta concentração natural de zinco (Zn) no tecido das ostras.

Os resultados deste trabalho indicam a necessidade do monitoramento das áreas de cultivo e de um plano para controlar poluição das águas dos seis municípios do entorno da Baía da Babitonga, envolvendo saneamento básico, tratamento de efluentes industriais e destino adequado de resíduos sólidos, entre outros cenários.

\section{ABSTRACT}

\section{EVALUATION OF HEAVY METAL CONCENTRATION IN TISSUE OF Crassostrea gigas (MOLUSCA, BIVALVE) OYSTERS CULTIVATED IN BABITONGA BAY, NORTH COAST OF SANTA CATARINA STATE, BRAZIL}

This work had as objective to evaluate the heavy metal concentration in the Crassostrea gigas oysters cultivated in 4 sites of Babitonga Bay, North seaside of Santa Catarina, Brazil. To do so, structures of cultivation were installed in each site in the long line system. During one year, samplings were obtained to analyze water physical-chemical parameters every 15 days and chemical analysis every 2 months, evaluating heavy metals like arsenic, cadmium, lead, copper, and zinc in the sediment, water and oysters' tissue. The results in relation to the heavy metals, showed values above to the quantity allowed by the Brazilian legislation to arsenic, nickel, copper, cadmium and zinc during summer. Although it was observed the presence of significant amounts of zinc in all points along the year. It was verified that the oysters presented assimilation of contaminants indicating the bioavailability in the environment, however, it was also observed the capacity of self-depuration of such organisms.

KEY-WORDS: MARINE AQUACULTURE; Crassostrea gigas; HEAVY METALS.

\section{REFERÊNCIAS}

1 AOAC. Association of Official Analytical Chemists. Official methods of AOAC International. $17^{\text {th }}$ ed. Washington, DC., 2000. chap. 9. p. 19-22.

2 AZEVEDO, F. A; CHASIN, A. M. Metais: gerenciamento da toxicidade. São Paulo: Editora Atheneu, 2003. 554 p.

3 BRASIL. Conselho Nacional do Meio Ambiente. Resolução CONAMAn. 20, de 18 de julho de 1986. Classifica segundo seus usos preponderantes as águas doces e salinas do Território Nacional. Diário Oficial [da] República Federativa do Brasil, Brasília, 12 fev. 1986, p. $2198-2199$. 
4 BRASIL. Portaria SVS/MS n‥685, de 27 de agosto de 1998. Regulamento técnico de princípios gerais para o estabelecimento de níveis máximos de contaminantes químicos em alimentos e seu anexo: limites máximos de tolerância para contaminantes inorgânicos. Diário Oficial [da] República Federativa do Brasil, Brasília, 28 ago. 1998. Seção 1.

5 CETESB. Companhia de Tecnologia de Saneamento Ambiental. Sistema Estuarino de Santos e São Vicente. São Paulo, 2001. 178 p.

6 FATMA/GTZ. Fundação do Meio Ambiente. Relevância de parâmetros de qualidade das águas aplicadas a águas correntes. Florianópolis, 1999.

7 GESAMP. Join Group of Experts on the Scientific Aspects of Marine Pollution. The state of the marine environment. [s.I.]: UNEP, 1990. (Regional Seas Reports and Studies, 115).

8 GOUDIE, A.S. The human impact: man's role in environmental change. Cambridge, MA.: Mit Press, 1989.316 p.

9 IBAMA. Instituto Brasileiro do Meio Ambiente e dos Recursos Naturais Renováveis. Proteção e controle de ecossistemas costeiros: manguezal da baía da Babitonga. Brasília,1998. 145 p. (Série Estudos: Pesca, 25).

10 LABORATÓRIO DE GEOPROCESSAMENTO E SENSORIAMENTO REMOTO. Prefeitura Municipal de Joinville. Secretaria do Meio Ambiente. Mapa de localização da baía da Babitonga. Joinville, 2002. 3 mapas: color. Escala: 1:100.000. Material cartográfico.

11 NEFF, J. M. Ecotoxicology of arsenic in the marine environment. Environmental Toxicology and Chemistry, v.16, n. 5, p. 917-927, 1997.

12 OliVEIRA NETO, F.M. Diagnóstico do cultivo de moluscos em Santa Catarina. Florianópolis: Epagri, 2005.67 p.

13 PEREIRA, S.A.; NASCIMENTO, I.A.; SMITH, D.H.; LEITE, M.B.N.L.; DE ARAÚJO, M.M.S; SILVA, M.A. The combined effects of temperature and metals copper, zinc and mercury on the embryological development of the mangrove oyster, Crassostrea rhizophorae. Ecotoxicology and Environmental Restoration, v. 1, n. 1, p. 21-32, 1994.

14 PEREIRA, A.; TEIXEIRA, A.L.; POLI, C. R.; BROGNOLI, F.F.; SILVA, F.C. da; RUPP, G.S.; SILVEIRA, JR. N.; ARAÚJO, S. C. Biologia e cultivo de ostras. Florianópolis: UFSC. 1998. 70 p.

15 RAND, G. M.; PETROCELLI, S. R. Fundamentals of aquatic toxicology: methods and applications. New York: Hemisphere Pub., 1985. 666 p.

16 TURECK, C. R.; OLIVEIRA, T.N.de. Sustentabilidade ambiental e maricultura. Revista Saúde e Meio Ambiente, Joinville, v.4, n.2, p 22- 26, 2003

17 UNESCO. United Nations Educational, Scientific and Cultural Organization. The international mussel watch. A global assessment of environmental levels of chemical contaminants. [s.I.]: IOC, 1992.

18 USEPA. Environmental Protection Agency. Monitoring guidance for the National Estuary Program. Washington,1992. (EPA 842-B-92-004).

19 WOLF, D. A. Zinc in aquatic animals. Baltimore: University Park Press, 1979. p. 87-122

\section{AGRADECIMENTOS}

Ao Fundo de Apoio a Pesquisas (FAP) Univille pelo financiamento do projeto, ao Laboratório de Moluscos Marinhos da Universidade Federal de Santa Catarina pelas sementes das ostras, às Associações de Maricultores de São Francisco do Sul parceiras no projeto, aos estagiários e colaboradores pela dedicação e à Emiliana Tiboni do Ceppa (UFPR) pelo carinho e atenção. 